\title{
$\lambda$-Carrageenan oligosaccharides elicit reactive oxygen species production resulting in mitochondrial-dependent apoptosis in human umbilical vein endothelial cells
}

\author{
HAI-MIN CHEN ${ }^{1}$, XIAO-JUN YAN ${ }^{1}$, TING-YAN MAI ${ }^{1}$, FENG WANG $^{2}$ and WEI-FENG XU ${ }^{2}$ \\ ${ }^{1}$ Ningbo University, Key Laboratory of Applied Marine Biotechnology, Ministry of Education, Ningbo, Zhejiang 315211; \\ ${ }^{2}$ Department of Clinical Laboratory, Lihuili Hospital of Ningbo Medical Center, Ningbo, Zhejiang 315041, P.R. China
}

Received June 10, 2009; Accepted August 7, 2009

DOI: 10.3892/ijmm_00000295

\begin{abstract}
Previous studies have shown that highly sulfated $\lambda$-carrageenan oligosaccharides $(\lambda$-CO) possess an antiangiogenetic effect, while high concentrations of $\lambda$-CO present a cytotoxic effect towards human umbilical vein endothelial cells (HUVECs). The aim of this study was to explore the underlying mechanism of $\lambda$-CO on inhibiting cell proliferation. $\lambda$-CO elicited reactive oxygen species (ROS) production with concentrations at 0.8 and $1 \mathrm{mg} / \mathrm{ml}$, and this event was accompanied by the increase of early apoptotic cells, nuclear morphology changes and cell cycle arrest at the $\mathrm{S}$ and $\mathrm{G}_{2} / \mathrm{M}$ phases. However, prevention of oxidative stress by $\mathrm{N}$-acetyl-L-cysteine (NAC) could abolish the effect of $\lambda$ $\mathrm{CO}$ on these events. Yet, $\lambda$-CO induced a depolarization of mitochondrial transmembrane potential. At $0.8 \mathrm{mg} / \mathrm{ml}, \lambda-\mathrm{CO}$ induced up-regulation of p53 and Bax, down-regulation of Bcl-2 and activation of caspase- 9 and -3 . These results suggest that exposure to a high concentration of $\lambda$-CO activates the mitochondrial-mediated apoptotic pathway and cell cycle arrest by generation of ROS.
\end{abstract}

\section{Introduction}

Carrageenans are high molecular weight, sulfated polygalactans derived from several species of red seaweeds. The most common forms of carrageenan are $\lambda, \kappa$, and $\iota(1) . \lambda$-carrageenan has a characterized structure. It contains alternating disaccharide units composed of D-galactose-2-sulfate and D-galactose-

Correspondence to: Professor Xiao-Jun Yan, Ningbo University, Key Laboratory of Applied Marine Biotechnology, Ministry of Education, Ningbo, Zhejiang 315211, P.R. China

E-mail: xiaojunyan@hotmail.com

Abbreviations: HUVECs, human umbilical vein endothelial cells; $\lambda$-CO, $\lambda$-carrageenan oligosaccharides; ROS, reactive oxygen species; MMP, mitochondrial membrane potential

Key words: $\lambda$-carrageenan oligosaccharides, human umbilical vein endothelial cells, reactive oxygen species, apoptosis 2,6-disulfate (2). Since $\lambda$-carrageenan contains a high degree of sulfate substitution in its structure (35\% ester sulfate content), it has been found to show biological activities such as antitumor, immunomodulation (3) and antiviral (4) and inhibiting tumor metastasis (5), especially the low molecular weight oligosaccharides. In our previous work, we also found that these oligosaccharides inhibit angiogenesis by blocking the endothelial cell invasion and migration at a relatively low concentration range $(60-250 \mu \mathrm{g} / \mathrm{ml})(6)$. However, $\lambda$-carrageenan has long been used as a typical inflammatory agent for establishing inflammatory models, such as pain models, paw oedema, arthritis, pleurisy or ulceration. Some reports also suggest that the degraded carrageenan (poligeenan), which is manufactured by the deliberate acid hydrolysis of carrageenan at high temperature $\left(>80^{\circ} \mathrm{C}\right)$, is the prime cause for inducing ulceration $(7,8)$. In our previous research, we observed that certain concentrations of $\lambda$-carrageenan oligosaccharides $(\lambda$ $\mathrm{CO})(>250 \mu \mathrm{g} / \mathrm{ml})$ inhibited cell survival, especially at a high concentration $(>800 \mu \mathrm{g} / \mathrm{ml})$ and the viability of human umbilical vein endothelial cells (HUVECs) was inhibited $>50 \%$ (6). Whether the high concentration of degraded $\lambda$-CO will disturb their therapeutic potential and how these oligosaccharides exert their cytotoxic effect is worth evaluating.

\section{Materials and methods}

Materials. $\lambda$-CO were self-prepared, as previously reported (6). JC-1(5,5',6,6'-tetrachloro-1,1',3,3'-tetraethylbenzimidazolylcarbocyanine iodide), N-acetyl-Lcysteine (NAC), 2',7'-dichlorofluorescein diacetate (DCFH-DA), Hoechst 33258 were obtained from Sigma (Shanghai, China). Annexin V-FITC apoptosis detection kit, Cycle Test ${ }^{\mathrm{TM}}$ Plus DNA reagent kit and FITC-conjugated monoclonal active caspase-3 antibody apoptosis kit were purchased from BD Biosciences Pharmingen (San Jose, CA, USA). CaspaTag ${ }^{\mathrm{TM}}$ caspase-9 in situ assay kit was purchased from Chemicon International, Inc. (Billerica, MA, USA). Mouse monoclonal antibodies specific for Bcl-2 (1:300) and $\beta$-actin $(1: 1,000)$, rabbit polyclonal antibodies specific for Bax (1:400) and p53 (1:500), horseradish peroxidase-conjugated goat anti-mouse or anti-rabbit secondary antibody $(1: 2,000)$ and ECL detection kit were obtained from Santa Cruz Biotechnology Inc. (Santa Cruz, CA, USA). 
Cell culture. HUVECs were obtained from ATCC (Rockville, MD, USA). Cells were cultured in Dulbecco's Modified Eagle's Medium (DMEM) supplemented with $10 \%$ newborn calf serum in a humidified atmosphere of $95 \%$ air and $5 \% \mathrm{CO}_{2}$ at $37^{\circ} \mathrm{C}$ in a carbon dioxide incubator.

Determination of reactive oxygen species (ROS) generation by flow cytometry. Intracellular ROS was detected by means of an oxidation-sensitive fluorescent probe 2',7'-dichlorofluorescein diacetate (DCFH-DA). After treatment, cells were washed twice in phosphate-buffered saline (PBS), followed by incubating with $20 \mathrm{mmol} / 1 \mathrm{DCFH}-\mathrm{DA}$ at $37^{\circ} \mathrm{C}$ for $45 \mathrm{~min}$. DCF fluorescence (excitation, $485 \mathrm{~nm}$ and emission, $525 \mathrm{~nm}$ ) was detected by FACSCalibur flow cytometry (Becton Dickinson). For each sample 10,000 events were collected.

Annexin V-FITC and propidium iodide (PI) double staining. AnnexinV-FITC/PI double stain assay was performed to examine $\lambda$-CO-induced apoptosis. Following the manufacturer's instructions, HUVECs treated with $\lambda$-CO in the presence or absence of NAC were washed and resuspended in binding buffer prior to the addition of FITC-labeled annexin V and PI for $10 \mathrm{~min}$. Suspensions were immediately analyzed by flow cytometry. Early apoptotic cells were defined as FITC $^{+} / \mathrm{PI}^{-}$ cells.

Determination of nuclear condensation by Hoechst staining. After treated with $\lambda$-CO, cells were washed in ice-cold PBS twice, fixed with $75 \%(\mathrm{v} / \mathrm{v}$ ) cold methanol for $30 \mathrm{~min}$ and then incubated with Hoechst 33258 for $45 \mathrm{~min}$ in the dark. Cells were then rinsed with PBS, mounted on glass microscopic slides in $50 \%$ glycerol, and examined under a fluorescent microscope.

Cell cycle analysis. Cells were treated with samples at different concentrations. Cell cycle analysis was performed with PI stained cellular DNA after debris removal as previously described (9). Flow cytometric analyses were performed on a FACSCalibur flow cytometry at $488 \mathrm{~nm}$ excitation and $620 \mathrm{~nm}$ emission wavelengths. Resulting histograms were analyzed by determining the percentage of cells in each phase with the ModFit LT software (Verity Software House, Inc., Topsham, ME, USA).

Determination of the mitochondrial membrane potential by JC-1 fluorescence. The mitochondrial membrane potential (MMP) was measured with the lipophilic cationic probe JC-1. HUVECs treated with/without $\lambda$-CO were incubated with $2.5 \mu \mathrm{g} / \mathrm{ml} \mathrm{JC}-1$ in the dark for $20 \mathrm{~min}$ at $37^{\circ} \mathrm{C}$. Cells were washed twice in PBS, resuspended in $400 \mu 1 \mathrm{PBS}$ and analyzed by flow cytometry. A 488-nm filter was used for excitation of JC-1. Emission filter of 535 and $595 \mathrm{~nm}$ were used to quantify the population of HUVECs with green (JC-1 monomer) and orange (JC-1 aggregates) fluorescence, respectively. The percentage of the population stained green (FL-1) and orange (FL-2) were measured by histograms. In addition, all samples were observed by fluorescence microscopy to confirm JC-1 labeling patterns.

Western blot analysis. Cells treated with $0.8 \mathrm{mg} / \mathrm{ml} \lambda$-CO for $0,12,24,36 \mathrm{~h}$ were harvested and lysed in $200 \mu \mathrm{l}$ ice-cold

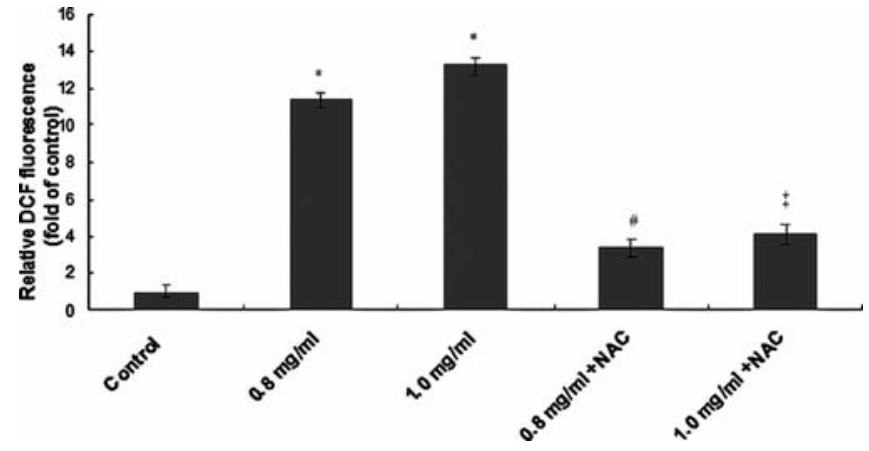

Figure 1. $\lambda$-CO induced intracellular ROS in HUVECs. Cells were treated with or without $\lambda$-CO for $24 \mathrm{~h}$, and incubated with DCFH-DA for $45 \mathrm{~min}$ in the dark. ROS level was analyzed immediately by FACS. The fluorescence intensity of untreated control cells was defined as $100 \%$ and the changes in cell fluorescence relative to the control was calculated $(n=3$, mean $\pm S D)$. ${ }^{*} \mathrm{P}<0.01$ compared with control, ${ }^{*} \mathrm{P}<0.01$ compared with $0.8 \mathrm{mg} / \mathrm{ml} \lambda$-CO, ${ }^{\ddagger} \mathrm{P}<0.01$ compared with $1.0 \mathrm{mg} / \mathrm{ml} \lambda$-CO.

lysis buffer. The soluble protein concentrations were determined by BCA assay. Protein was subjected to SDS-PAGE and transferred to a PVDF membrane. After blocking with 5\% nonfat milk, the membrane was incubated overnight at $4^{\circ} \mathrm{C}$ with primary antibodies (anti-Bcl-2, anti-Bax and anti-p53 antibody). After washing, blots were incubated with HRPconjugated secondary antibody. Chemiluminescence was used to visualize the bands. The net intensity of bands was calculated to analyze the relative content of Bcl-2, Bax, and p53.

Active caspase- 9 detection. The activities of caspase- 9 were measured by CaspaTag ${ }^{\mathrm{TM}}$ caspase-9 in situ assay kit. According to the manufacturer's instruction, harvested cells treated with or without $\lambda$-CO were incubated with $10 \mu \mathrm{l}$ of freshly prepared FLICA reagent for $1 \mathrm{~h}$ at $37^{\circ} \mathrm{C}$ in $5 \% \mathrm{CO}_{2}$ in the dark. Then, cells were washed twice in PBS, resuspended in $400 \mu 1$ wash buffer and analyzed by flow cytometry.

Active caspase-3 detection. Caspase-3 activity was detected by using FITC-conjugated monoclonal active caspase- 3 antibody apoptosis kit. Cells at a concentration of $1 \times 10^{6}$ cells $/ \mathrm{ml}$ were incubated with FITC-conjugated caspase- 3 antibody $(10 \mu \mathrm{g} / \mathrm{ml})$ for $20 \mathrm{~min}$ in the dark and rinsed twice in PBS. Cells were measured by flow cytometry after resuspended in $1 \mathrm{ml}$ of PBS.

Statistical analysis. Data were expressed as means \pm standard deviation (SD) of three separate experiments. Significance of differences among groups was determined using Student's t-test. Statistical significance was defined as $\mathrm{P}<0.05$.

\section{Results}

Effects of $\lambda$-CO on intracellular ROS level. In our previous studies, we found that high concentrations of $\lambda$-CO showed an inhibitive growth effect on HUVECs (at $1 \mathrm{mg} / \mathrm{ml}$, the inhibitory ratio reached upto $60.23 \%$ ) (6). Considering that oxidative stress is an important factor in causing cell death (10) and hyperglycaemia also elicits oxidative stress in cells (11) we firstly explored the effects of $\lambda$-CO on the accumulation 

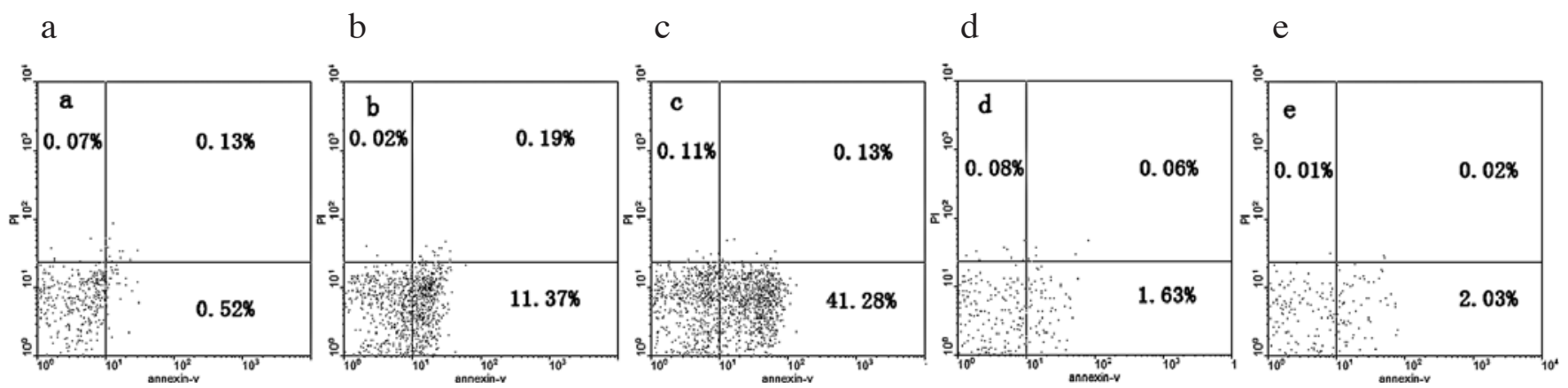

Figure 2. Apoptosis detection in HUVECs using the annexin V assay. Cells were exposed to (a) vehicle; (b) $0.8 \mathrm{mg} / \mathrm{ml} \lambda$-CO; (c) $1 \mathrm{mg} / \mathrm{ml} \lambda$-CO; (d) $0.8 \mathrm{mg} / \mathrm{ml}$ $\lambda$-CO+10 mmol/1 NAC; (e) $1 \mathrm{mg} / \mathrm{ml} \lambda$-CO+10 mmol/1 NAC. Apoptotic cells were determined after $24 \mathrm{~h}$. Cells per treatment condition $(10,000)$ were analyzed by flow cytometry. In each plot, the Annexin V-FITC+PI- denotes early apoptotic cells (lower right quadrant).

Table I. Effect of $\lambda$-CO on cell cycle distribution in HUVECs (\%).

\begin{tabular}{|c|c|c|c|c|}
\hline & & $\mathrm{G}_{1}$ phase & S phase & $\mathrm{G}_{2} / \mathrm{M}$ phase \\
\hline Control & & $67.14 \pm 1.73$ & $22.62 \pm 4.07$ & $10.24 \pm 2.69$ \\
\hline \multirow[t]{4}{*}{$\lambda$-CO treated groups } & $0.8 \mathrm{mg} / \mathrm{ml}$ & $46.35 \pm 5.09$ & $30.96 \pm 2.15$ & $22.69 \pm 4.21$ \\
\hline & $1.0 \mathrm{mg} / \mathrm{ml}$ & $23.61 \pm 2.83$ & $42.36 \pm 0.95$ & $34.13 \pm 1.84$ \\
\hline & $0.8 \mathrm{mg} / \mathrm{ml}+\mathrm{NAC}$ & $58.85 \pm 4.78$ & $29.91 \pm 3.09$ & $11.27 \pm 1.55$ \\
\hline & $1.0 \mathrm{mg} / \mathrm{ml}+\mathrm{NAC}$ & $63.11 \pm 3.85$ & $25.85 \pm 3.76$ & $11.04 \pm 2.01$ \\
\hline
\end{tabular}

of intracellular ROS using the fluorescent probe DCFH-DA. The results show that incubation with $\lambda$-CO for $24 \mathrm{~h}$ leads to a remarkable increase in intracellular ROS compared with control. At a dose of 0.8 and $1.0 \mathrm{mg} / \mathrm{ml} \lambda$-CO, intracellular ROS levels were 11.4 and 13.2 times that of the control group, respectively (Fig. 1). The addition of a known antioxidant $\mathrm{N}$-acetylcysteine largely blocked the production of ROS, which reveals that $\lambda$-CO treatment can trigger ROS production.

Dose response of $\lambda$-CO-induced apoptosis and effect of NAC on $\lambda$-CO-induced apoptosis. Apoptosis was analyzed by annexin $\mathrm{V}$ assay, which detects the arrangement of phosphatidylserine from the inner to the outer plasma membrane leaflet during early stages of apoptosis (12). The increase in the number of apoptitic cells is shown in Fig. 2. Treatment of HUVECs with 0.8 and $1.0 \mathrm{mg} / \mathrm{ml} \lambda$-CO for $24 \mathrm{~h}$ induced a dose-dependent increase in the percentage of apoptotic cells compared with controls. At $1.0 \mathrm{mg} / \mathrm{ml}, 41.11 \%$ of cells were apoptotic. Interestingly, we observed that $\lambda$-CO-induced apoptosis was remarkably abolished by pretreatment with NAC. The apoptotic ratios decreased to $1.14 \pm 0.78$ and $1.52 \pm 0.92 \%$ (Fig. $2 \mathrm{~d}$ and e). This result clearly confirms that ROS production is the pivotal process in $\lambda$-CO-induced HUVEC apoptosis.

To further explore whether the apoptotic effect of $\lambda-\mathrm{CO}$ influences the morphology of cell nuclei, the changes of nuclear features were detected by Hoechst 33258 (Fig. 3). Visualization of fluorescence revealed typical chromatin condensation after $0.8 \mathrm{mg} / \mathrm{ml} \lambda$-CO treatment. When HUVECs were treated with $1 \mathrm{mg} / \mathrm{ml} \lambda-\mathrm{CO}$, the margination in some

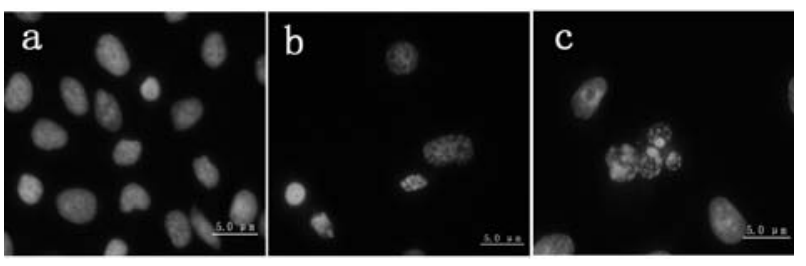

Figure 3. Nuclear morphological changes of HUVECs treated with $\lambda$-CO. Cells were incubated with or without $\lambda-\mathrm{CO}$ for $24 \mathrm{~h}$ and observed by fluorescence microscopy after Hoechst 33258 staining. (a) Untreated cells; (b) $0.8 \mathrm{mg} / \mathrm{ml} \lambda$-CO; (c) $1 \mathrm{mg} / \mathrm{ml} \lambda$-CO.

cells and apoptotic body-like structures appeared, which indicates that $\lambda$-CO-induced apoptosis of HUVECs eventually resulted in the fragmentation of nuclei.

Effect of $\lambda$-CO on cell cycle distribution of HUVECs. FACS analysis was performed to determine the effect of $\lambda$-CO on cell cycle. $\lambda$-CO induced the increase of cells in the $\mathrm{S}$ and $\mathrm{G}_{2} / \mathrm{M}$ phase, concomitant with a decrease in $\mathrm{G}_{0} / \mathrm{G}_{1}$ in a dosedependent manner, especially the high dose of $1 \mathrm{mg} / \mathrm{ml} \lambda$-CO improved the population of cells in $\mathrm{G}_{2} / \mathrm{M}$ by $\sim 25 \%$ (Table I). Yet, the cells accumulated in the $\mathrm{S}$ phase do not suggest that $\lambda$ $\mathrm{CO}$ induces $\mathrm{S}$ phase arrest because cells in this phase could have entered the $G_{2} / M$ partly to increase $G_{2} / M$ content. In contrast, the decrease of $\mathrm{G}_{0} / \mathrm{G}_{1}$ population revealed obviously that the $\mathrm{G}_{2} / \mathrm{M}$ phase was arrested. These results suggest that $\lambda$-CO treatment prevented or slowed down the synthesis of DNA, and the $\mathrm{G}_{2} / \mathrm{M}$ checkpoint blocked the entry of cells into mitosis when DNA was damaged. However, pretreated with 
A
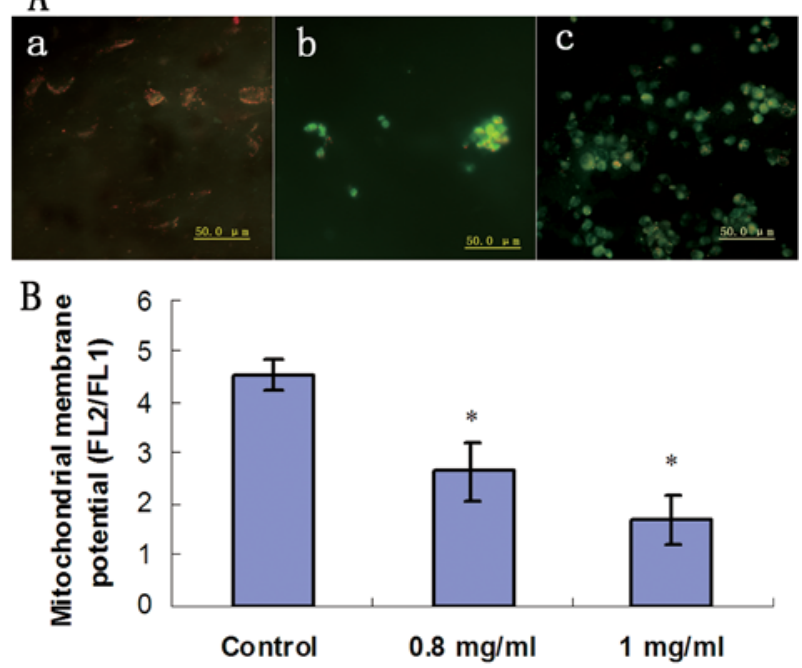

Figure 4. Effect of $\lambda$-CO on mitochondrial membrane potential in HUVECs. Cells were incubated with (a) vehicle (b) $0.8 \mathrm{mg} / \mathrm{ml}$ (c) $1.0 \mathrm{mg} / \mathrm{ml}$ of $\lambda$-CO for $24 \mathrm{~h}$ and then the alteration of mitochondrial membrane potential $(\Delta \psi \mathrm{m})$ was determined by loading with JC-1 $(2.5 \mu \mathrm{g} / \mathrm{ml})$ for $20 \mathrm{~min}$. (A) Samples were observed by fluorescence microscopy. The fluorescent images in the green and red channels were captured, and images under the two channels were merged together. (B) The percentages of cells containing polarized or depolarized mitochondria were determined by histogram analysis of the ratio of the two fluorescence intensities (FL-2/FL-1) obtained by flow cytometry. Data represent means of three independent experiments.

NAC profoundly reduced the percentage of cells in the $\mathrm{S}$ and $\mathrm{G}_{2} / \mathrm{M}$ phases, which indicates that the antioxidant property of NAC repairs DNA damage triggered by $\lambda$-CO, or stops $\lambda$-CO from injuring DNA. Interestingly, we observed a marked increase of $\mathrm{G}_{1}$ population after the addition of NAC, and this result was also found by Menon et al, who reported that NAC inhibits cell progression from $\mathrm{G} 1$ to $\mathrm{S}$ phase, causing G1 arrest (13).

Effects of $\lambda$-CO on MMP. The loss of MMP $(\Delta \psi \mathrm{m})$ is a hallmark for apoptosis. Tan et al suggested that the mitochondrial electron transport chain is a major source of cellular ROS (14). To determine whether $\lambda$-CO-induced apoptosis involves the disruption of integrity of the mitochondrial membrane, MMP was measured using JC-1. Fig. 4 shows fluorescence microscopy of JC- 1 staining of HUVECs with or without $\lambda$ $\mathrm{CO}$ treatment. High mitochondrial polarization is indicated by red fluorescence and depolarized regions are indicated by green fluorescence (15). In Fig. 4a, most cells without $\lambda$-CO treatment show red fluorescence in mitochondrion, indicating integrity of the mitochondrial membrane. However, $\lambda$-CO treatment induced perturbation in the function of the mitochondrial membrane, as indicated by the red to green color switch in a great number of HUVECs. Flow cytometry quantitative analysis performed on cells after JC-1 labeling, clearly shows that $\lambda$-CO induced alteration in the mitochondrial membrane 2.7-fold compared with untreated cultures (Fig. 4B). Therefore, these results indicate that $\lambda$-CO treatment induce the disruption of integrity of mitochondrial membrane in HUVECs, which may subsequently involve the release of ROS and eventually induce apoptosis of cells.
A

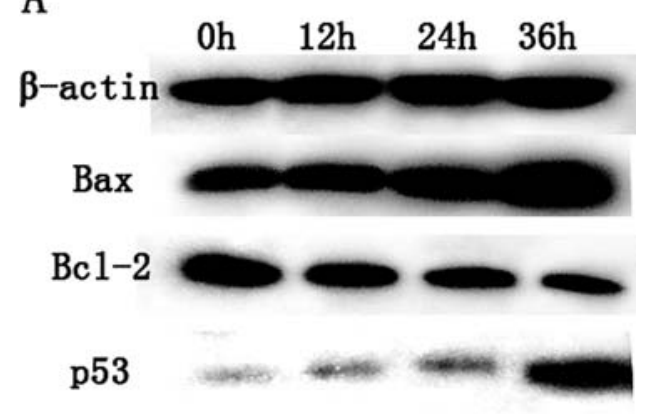

B

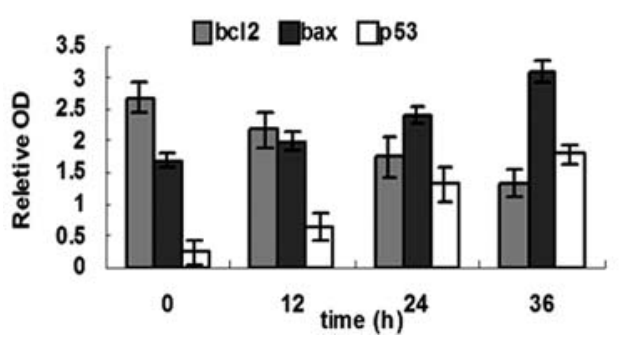

Figure 5. Western blot analysis of Bcl-2, Bax, and p53 expression in HUVECs. (A) HUVECs were treated with $\lambda$-CO for 12, 24, 36 h. Cell lysates were prepared and immunoblotted as described in Materials and methods. The levels of $\beta$-actin served as a loading control. (B) Densitometric quantification of these proteins was measured by Kodak digital science software. $n=3$; mean $\pm \mathrm{SD} ;{ }^{*} \mathrm{P}<0.05$ compared with control group.

Effects of $\lambda$-CO on the expression and activation of apoptoticrelated proteins. The protein expression of some apoptosis associated genes, including Bcl-2, Bax and p53 were measured with the aim of assessing possible involvement of $\lambda$-CO in mitochondrial-mediated apoptosis. p53 is crucial to the apoptotic pathway which is found to trigger programmed cell death in response to DNA damage (16). Results from Western blotting show that after treated with $\lambda$-CO, level of p53 expression was up-regulated (Fig. 5). In control group, p53 level was low and undetectable, while $\lambda$-CO induced a timedependent accumulation of $\mathrm{p} 53$. Since $\mathrm{p} 53$, in response to cellular stress, has been linked to mitochondrial-mediated apoptosis by the transcriptional activation of its target genes, including pro-apoptotic Bax gene and anti-apoptotic Bcl-2, we examined the expression of these two proteins. The significant decrease of $\mathrm{Bcl}-2$ and increase of Bax protein were achieved from 12 to $36 \mathrm{~h}$. We measured the optical density of these bands and found that the Bcl-2/Bax ratio decreased. After $36 \mathrm{~h}$ treatment of $\lambda$-CO, the ratio of $\mathrm{Bcl}-2 / \mathrm{Bax}$ expression decreased $\sim 3$-fold compared with the control group (Fig. 5B).

With the mitochondrial membrane potential destroyed by ROS, the permeability transition pore will open and intermembrane proteins are released from the mitochondria, which in turn activates the downstream caspase-9 (17). The level of active caspase- 9 was detected by flow cytometry. Results show that cells with active caspase- 9 increased significantly after treatment with $\lambda$-CO (Fig. 6). Cells with positive active caspase- 9 were $33.24 \pm 4.62$ and $71.10 \pm 4.89 \%$ for 0.8 and $1.0 \mathrm{mg} / \mathrm{ml} \lambda$-CO treatment, respectively. Caspase- 3 is one of the key executioners of apoptosis (18). After treated with $\lambda$-CO for 24 and $48 \mathrm{~h}$, HUVECs with activated caspase- 3 were 


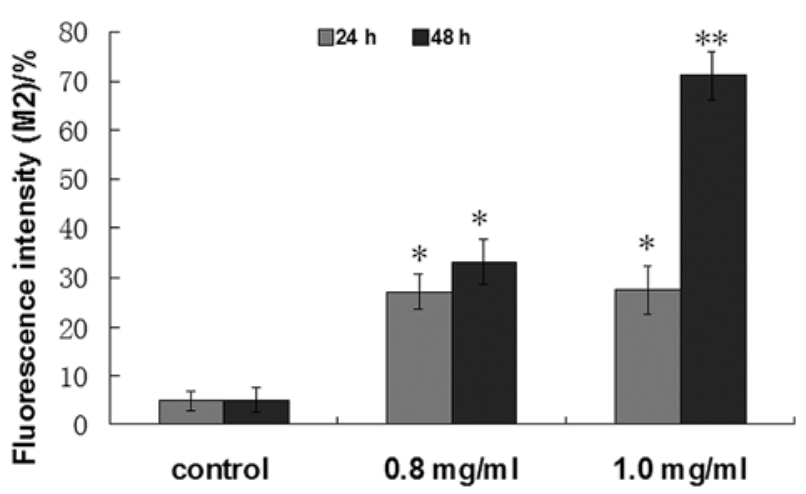

Figure 6. Levels of activated caspase-9. After treatment with 0.8 and 1 $\mathrm{mg} / \mathrm{ml} \lambda$-CO for 24 or $48 \mathrm{~h}$, activated caspase- 9 was detected using a CaspaTag caspase-9 in situ assay kit and analyzed by flow cytometry. The representative histograms from three independent experiments are shown. ${ }^{*} \mathrm{P}<0.05,{ }^{* *} \mathrm{P}<0.01$ compared with control.

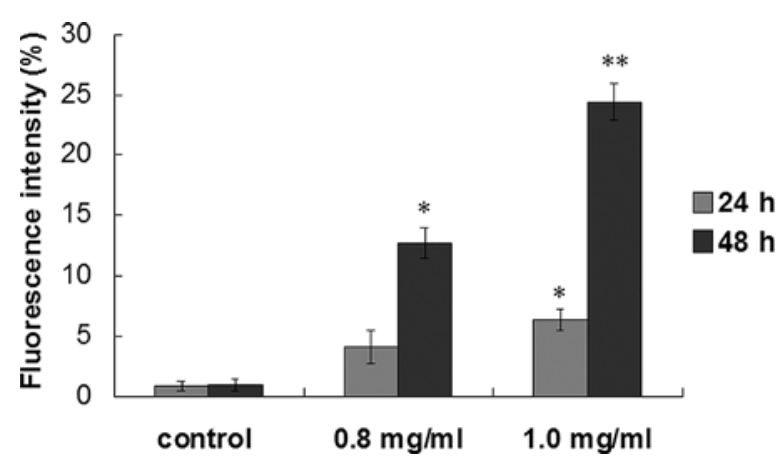

Figure 7. Levels of activated caspase-3. After treated with different concentrations of $\lambda$-CO for 24 or $48 \mathrm{~h}$, activated caspase- 3 was detected using FITC-conjugated monoclonal active caspase- 3 antibody apoptosis kit and analyzed by flow cytometry. The representative histograms from three independent experiments are shown. ${ }^{*} \mathrm{P}<0.05,{ }^{* *} \mathrm{P}<0.01$ compared with control.

increased from only $0.87 \pm 0.4$ and $0.97 \pm 0.5 \%$ in the control group to $6.30 \pm 0.9$ and $24.39 \pm 1.6 \%$ in the $1 \mathrm{mg} / \mathrm{ml}$ group (Fig. 7). Collectively, these results suggest that exposure of $\lambda$-CO led to the activation of the mitochondria-mediated apoptotic pathway including up-regulation of Bax, downregulation of the $\mathrm{Bcl}-2$ gene associated with activation of caspase- 9 and -3 .

\section{Discussion}

Considering our previous studies, $\lambda$-carrageenan with a molecular weight of $\sim 3,350$ showed anti-angiogenesis activity at a low concentration $(<250 \mu \mathrm{g} / \mathrm{ml})$. At a high concentration from 0.5 to $1 \mathrm{mg} / \mathrm{ml}, \lambda$-CO exhibited an inhibitive effect on the proliferation of HUVECs. The question is, whether this kind of cell death will disturb or assist the application of $\lambda$-CO as an anti-angiogenesis agent. We performed this study in order to clarify the precise molecular mechanism involved in the inhibitory effect of $\lambda$-carrageenan on cell proliferation.

Our results clearly show that treatment of HUVECs with $\lambda$-CO elicited the production of ROS, evidenced by DCFH-DA fluorometric assay. The generated ROS may play a role in the inhibition of cell proliferation and the inducement of apoptosis

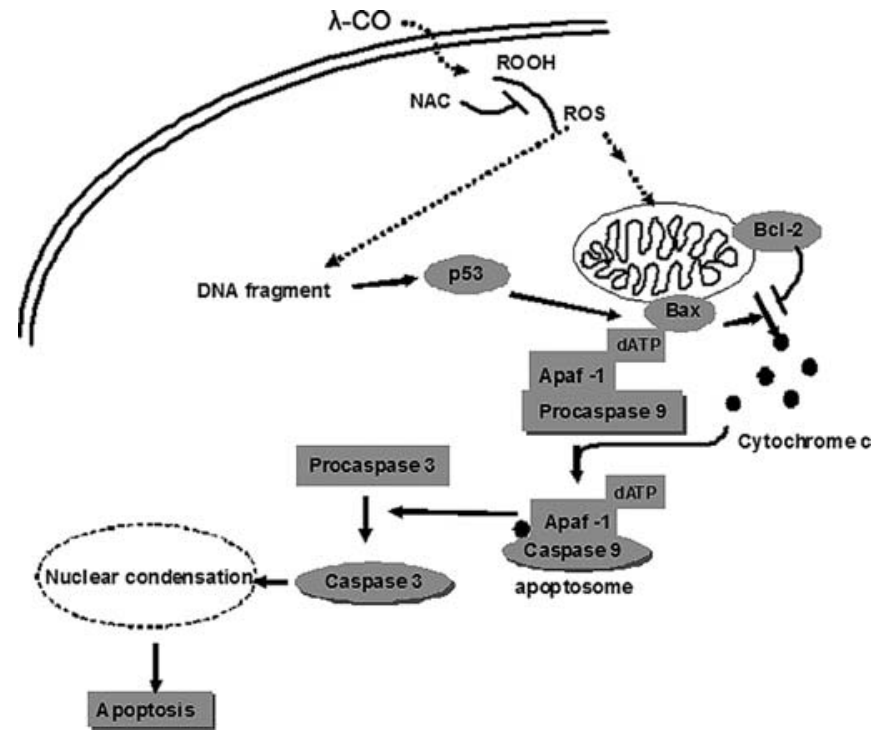

Figure 8. Model for the mechanism of apoptosis induced by $\lambda$-CO in HUVECs. Treatment of $\lambda$-CO elicits the initial generation of ROS which impairs mitochondrial function and destroys mitochondrial integrity. The loss of mitochondrial membrane potential may induce the cell mitochondria-mediated molecular pathway of apoptosis, observed as increasing level of Bax and decreasing level of $\mathrm{Bcl}-2$. Then the release of intermembrane proteins such as cytochrome c will interact with Apaf-1 to activate caspase-9 and ultimately activates downstream caspase-3. Caspase-3 activation led to DNA breakage, nuclear chromatin condensation, and cell apoptosis. As a consequence, nuclear lamins are cleaved, leading to apoptosis.

in HUVECs. Quenching ROS by NAC, an oxidant scavenger, not only suppressed the ROS generation, but also largely reversed the apoptosis caused by $\lambda$-CO. On the other hand, ROS may lead to free radical attack of membrane phospholipids and loss of MMP (19), inducing the activation of Bax, and active caspase-9, which ultimately activates the downstream caspase-3 (20,21). Caspase-3 activation led to DNA breakage and cell apoptosis. In our case, the apoptosis induced by $\lambda$-CO perfectly tallied with this procedure since we observed the loss of mitochondrial transmembrane potential, down-regulation of Bcl-2, up-regulation of Bax and enhanced active caspase- 9 and -3 . Therefore, it is confirmed that $\lambda$-COinduced cell death belongs to a typical mitochondrialmediated apoptotic pathway.

In the present study, HUVECs displayed an oxidative stress-induced cell cycle arrest. $\lambda$-CO-promoted cells continued through to the G1 phase and accumulated at the $\mathrm{S}$ and $\mathrm{G}_{2} / \mathrm{M}$ phases. In contrast, cells coincubated with $\lambda-\mathrm{CO}$ and NAC could go through the $\mathrm{G}_{2} / \mathrm{M}$ phase. These findings implicate that oxidative stress induced by $\lambda$-CO controls the cell cycle by attacking DNA or influencing the DNA synthesis rate, and eventually arrests cells at the $\mathrm{G}_{2} / \mathrm{M}$ phase. We examined the mechanism of dysregulated cell cycle arrest in HUVECs. It was observed that the p53 protein was up-regulated after exposure to $0.8 \mathrm{mg} / \mathrm{ml} \lambda$-CO. In response to ROS-induced DNA damage, p53 may also guide corrupt cells into apoptosis through mitochondrial mediated pathway by inducing expression of proapoptotic protein Bax.

Our previous study revealed that $\lambda$-CO exhibited antiangiogenesis efficacy at a concentration $<250 \mu \mathrm{g} / \mathrm{ml}$, while no cytotoxicity against HUVECs was observed (6). We believe 
that the anti-proliferation effect of high doses of $\lambda$-CO cooperates in reducing the proliferation of a network of blood vessels by promoting the apoptosis of vascular endothelial cells since in tumor tissue the vascular endothelial cells have abnormal growth (22).

Taken together, our study indicates that ROS mediates apoptosis in HUVECs caused by $\lambda$-CO, and that the mitochondria pathway is involved. Based on the results obtained to date, our working hypothesis (Fig. 8) is that the $\lambda$-CO triggered the generation of intracellular ROS, and the accumulation of ROS is an initiator of apoptosis by upregulating the expression of $\mathrm{p} 53$, which induced the decrease of MMP, and caused downstream caspase activation (including caspase-9 and -3).

\section{Acknowledgements}

This work was supported by grants from the National 863 Program (2007AA09Z412), K.C. Wong Magna Fund in Ningbo University, NSFC project (30800860), Program for Changjiang Scholars, Innovative Research Team in University (NO. IRT0734).

\section{References}

1. De Ruiter GA and Rudolph B: Carrageenan biotechnology. Trends Food Sci Tech 8: 389-395, 1997.

2. Borthakur A, Bhattacharyya S, Dudeja PK and Tobacman JK: Carrageenan induces interleukin- 8 production through distinct Bcl10 pathway in normal human colonic epithelial cells. Am J Physiol Gastrointest Liver Physiol 292: G829-G838, 2007.

3. Zhou GF, Sun YP, Xin H, Zhang Y, Li Z and Xu Z: In vivo antitumor and immunomodulation activities of different molecular weight lambda-carrageenans from Chondrus ocellatus. Pharmacol Res 50: 47-53, 2004.

4. Carlucci MJ, Scolaro LA and Damonte EB: Herpes simplex virus type 1 variants arising after selection with an antiviral carrageenan: Lack of correlation between drug susceptibility and syn phenotype. J Med Virol 68: 92-98, 2002.

5. Parish CR, Coombe DR, Jakobsen KB, Bennett FA and Underwood PA: Evidence that sulphated polysaccharides inhibit tumour metastasis by blocking tumour-cell-derived heparanases. Int J Cancer 40: 511-518, 1987.

6. Chen HM, Yan XJ, Lin J, Wang F and Xu WF: Depolymerized products of $\lambda$-carrageenan as a potent angiogenesis inhibitor. $J$ Agric Food Chem 55: 6910-6917, 2007.
7. Cohen SM and Ito NA: Critical review of the toxicological effects of carrageenan and processed eucheuma seaweed on the gastrointestinal tract. Crit Rev Toxicol 32: 413-444, 2002.

8. Wakabayaski K, Inagaki T, Fujimoto Y and Fukuda Y: Induction by degraded carrageenan of colorectal tumors in rats. Cancer Lett 4: 171-176, 1978.

9. Mertens-Talcott SU, Talcott ST and Percival SS: Low concentrations of quercetin and ellagic acid synergistically influence proliferation, cytotoxicity and apoptosis in MOLT-4 human leukemia cells. J Nutr 133: 2669-2674, 2003.

10. Ueda S, Masutani G, Nakamura G, Tanaka T, Ueno M and Yodoi F: Redox control of cell death. Antioxid Redox Signal 4: 405-514, 2002.

11. Zhao YL, Lu NH, Li HL and Zhang Y: High glucose induced human umbilical vein endothelial cell injury: involvement of protein tyrosine nitration. Mol Cell Biochem 311: 19-29, 2008.

12. Boersma AWM, Nooter K, Oostrum RG and Stoter G: Quantification of apoptotic cells with fluorescein isothiocyanatelabeled annexin $\mathrm{V}$ in Chinese hamster ovary cell cultures treated with cisplatin. Cytometry 24: 123-130, 1996.

13. Menon SG, Sarsour EH, Spitz DR, Higashikubo R, Sturm M and Zhang H: Redox regulation of the G1 to $S$ phase transition in the mouse embryo fibrolast cell cycle. Cancer Res 63: 2109-2117, 2003.

14. Tan S, Sagara Y, Liu Y, Maher P and Schubert D: The regulation of reactive oxygen species production during programmed cell death. Cell Biol 141: 1423-1432, 1998.

15. Salvioli S, Ardizzoni A, Franceschi C and Cossarizza A: JC-1, but not DiOC6(3) or rhodamine 123, is a reliable fluorescent probe to assess delta psi changes in intact cells: implications for studies on mitochondrial functionality during apoptosis. FEBS Lett 411: 77-82, 1997.

16. Oren M: Decision making by p53: life, death and cancer. Cell Death Differ 10: 431-442, 2003.

17. Kasahara Y, Iwai K, Yachie A, Ohta K, Konno A and Seki H: Involvement of reactive oxygen intermediates in spontaneous and CD95 (Fas/APO-1)-mediated apoptosis of neutrophils. Blood 89: 1748-1753, 1997.

18. Budihardjo I, Oliver H, Lutter M, Luo X and Wang X: Biochemical pathways of caspase activation during apoptosis. Annu Rev Cell Dev Biol 15: 269-290, 1999.

19. Petit PX, Lecoeur H, Zorn E, Dauguest C, Mignotte B and Gougeon ML: Alterations in mitochondrial structure and function are early events of dexamethasone-induced thymocyte apoptosis. J Cell Biol 130: 157-167, 1995.

20. Coffey RN, Watson RW and Fitzpatrick JM: Signaling for the caspases: their role in prostate cell apoptosis. J Urol 165: 5-14, 2001.

21. Wang $\mathrm{J}$ and Lenardo MJ: Roles of caspases in apoptosis, development, and cytokine maturation revealed by homozygous gene deficiencies. J Cell Sci 113: 753-757, 2000.

22. Jekunen A and Kairemo K: Inhibition of angiogenesis at endothelial cell level. Microsc Res Tech 60: 85-97, 2002. 